\title{
ESTUDO DA DISPERSÃO DE SURFACTANTES CATIÔNICOS COM DIFERENTES CABEÇAS HIDROFÍLICAS PARA FINS CATALÍTICOS
}

\author{
P. M. FUKUDA ${ }^{1}$, L. B. OLIVEIRA ${ }^{1}$, L. L. DA SILVA ${ }^{1}$, P. P. MODESTO NETO ${ }^{1}$ e D. \\ CARDOSO $^{1}$ \\ ${ }^{1}$ Universidade Federal de São Carlos, Departamento de Engenharia Química \\ E-mail para contato: dilson@ufscar.br
}

\begin{abstract}
RESUMO - O biodiesel é comumente produzido através da reação de transesterificação do óleo vegetal ou gordura animal, com um álcool de cadeia curta na presença de um catalisador básico, gerando ésteres e glicerol. A catálise heterogênea básica, quando utilizada nesta reação, apresenta vantagens como a possibilidade de reutilização do catalisador, simplicidade dos processos de separação e lavagem, além de não ser necessária a neutralização do biodiesel, porém estudos estão em desenvolvimento para melhorar a atividade, seletividade e estabilidade desse tipo de catalisador. Neste trabalho foram estudados os surfactantes catiônicos, fundamentais na síntese e na variação de propriedades estruturais das sílicas híbridas da Família M41S, já utilizada como catalisador heterogêneo nesta reação. Estes surfactantes sofrem o efeito hidrofóbico e formam micelas a partir de uma determinada concentração, chamada de Concentração Micelar Crítica (CMC). Identificou-se a CMC de dispersões contendo surfactantes catiônicos com cabeça hidrofílicas de diferentes tamanhos e com variação na temperatura $\left(25^{\circ} \mathrm{C}, 40^{\circ} \mathrm{C}\right.$ e $\left.50^{\circ} \mathrm{C}\right)$, por meio do método da condutividade elétrica. Os surfactantes utilizados possuem 16 carbonos em sua cauda hidrofóbica e se distinguem estruturalmente apenas por sua cabeça hidrofílica. O presente trabalho determinou correlações entre a temperatura e a CMC dos surfactantes apresentados, e verificou que alguns deles seguiam uma tendência em relação a CMC e volume da cabeça hidrofílica.
\end{abstract}

\section{INTRODUÇÃO}

A crescente tendência em relação a utilização de biocombustíveis e outras fontes alternativas de energia, se deve as reservas limitadas e não renováveis dos combustíveis de origem fóssil, bem como os impactos ambientais gerados por eles. Nessa perspectiva, o biodiesel se destaca no Brasil devido aos incentivos governamentais para sua produção e a disponibilidade de matéria-prima (Quintella, 2009). O biodiesel pode ser produzido utilizando a rota química, através da reação de transesterificação do óleo vegetal ou gordura animal, com um álcool de cadeia curta (usualmente, o metanol) na presença de um catalisador básico, gerando ésteres e glicerol (Georgogianni et al., 2009). Comumente a catálise homogênea é utilizada industrialmente e apresentam elevados rendimentos, no entanto a catálise heterogênea básica possui vantagens sobre a homogênea, como: possibilidade de reutilizar estes catalisadores, realização do processo de separação por uma simples operação unitária, 
simples processo de lavagem e a neutralização não ser necessária. Neste contexto, a busca por catalisadores heterogêneos básicos com alta atividade, estabilidade e seletividade tornou-se interessante (Fabiano, 2010; Silva, 2014; Campos, 2015; Araújo, 2013).

A CTA-MCM-41 é uma sílica híbrida utilizada como catalisador heterogêneo básico pelo grupo de pesquisa. Esta sílica pertence à Família M41S, que se caracteriza por seu arranjo hexagonal, poros unidimensionais (Grecco et al., 2013) e quando não calcinada tem sítios básicos do tipo silóxi $\left(\equiv \mathrm{SiO}^{-}\right)$na superfície e interior de seus mesoporos (Kubota et al., 2004). As sílicas híbridas com estrutura MCM-41 são sintetizadas em meio aquoso utilizando uma fonte de sílica, uma fonte de hidroxila e um surfactante catiônico. Entre os surfactantes catiônicos que podem ser utilizados na síntese o brometo de cetiltrimetilamônio (CTABr) é o mais utilizado. Estudos recentes do grupo de pesquisa do LabCat - UFSCar mostraram a influência da escolha de diferentes surfactantes catiônico nas propriedades estruturais da sílica híbrida (Fabiano, 2010; Araújo et al, 2013; Silva, 2014; Campos, 2015).

Os surfactantes são moléculas de caráter anfifílico, ou seja, possuem uma parte polar e outra apolar em sua estrutura. Devido a este caráter, os surfactantes quando em meio aquoso sofrem o efeito hidrofóbico, no qual a parte apolar tende a minimizar e a parte polar tende a maximizar o contato com a água (Oseliero Filho, 2013). Dessa forma, a partir de uma concentração mínima, característica de cada surfactante, são formadas as micelas e tal concentração mínima é chamada de Concentração Micelar Crítica (CMC) (Maniasso, 2001). A CMC pode ser determinada por diferentes métodos que analisam algumas propriedades físico-químicas das dispersões, como: tensão superficial, condutividade elétrica, solubilização, calorimetria, entre outras. Tais propriedades sofrem variações bruscas devido à formação de micelas. O método que analisa a condutividade elétrica possui algumas vantagens em relação aos demais, uma vez que apresenta custos relativamente baixos, alta precisão e rapidez na obtenção de resultados (Galgano, 2012). Além da CMC, existem outros parâmetros importantes em relação às dispersões de surfactantes, como a fração de contraíons dissociados na dispersão $(\alpha)$ e associados $(\beta)$. Os quais podem ser obtidos por meio do método de Frahm e são relacionados entre si, $(\alpha=1-\beta)$ (Galgano; El Seoud, 2010).

Nesse contexto, o trabalho teve como objetivo determinar a CMC (mmol/L) e a fração de contra-íons dissociados $(\alpha)$ pelo método da condutividade elétrica de dispersões contendo os surfactantes catiônicos de cauda cetil $\left(\mathrm{C}_{16}\right)$ e diferentes cabeças hidrofílicas a temperaturas distintas. Além de, identificar correlações entre a CMC obtida e o tamanho da cabeça hidrofílica dos surfactantes para cada temperatura avaliada.

\section{METODOLOGIA}

Os surfactantes catiônicos que foram avaliados nesse trabalho são os brometos de: cetiltrimetilamônio $(\mathrm{CTABr})$, cetiltrietilamônio $(\mathrm{C} 16 \mathrm{Et} 3 \mathrm{Br})$, cetiltripropilamônio $(\mathrm{C} 16 \mathrm{Pr} 3 \mathrm{Br})$, cetilpiridínio $(\mathrm{CPyBr})$ cetilalildimetilamônio $(\mathrm{CADMABr})$, cetilmetilimidazonio $(\mathrm{CMImBr})$, cetilvinilimidazonio (CVImBr), cetildimetiletilamônio $\quad(\mathrm{C} 16 \mathrm{Me} 2 \mathrm{Et} 1 \mathrm{Br}) \quad \mathrm{e}$ cetildietilmetilamônio (C16Me1Et2Br). A Tabela 1 apresenta as estruturas destes surfactantes. 
Tabela 1 - Estrutura dos surfactantes comerciais e os sintetizados no LabCat.

\begin{tabular}{|c|c|c|c|}
\hline Nomenclatura & Estrutura Molecular & Nomenclatura & Estrutura Molecular \\
\hline \multicolumn{2}{|c|}{ Comerciais } & \multicolumn{2}{|c|}{ Sintetizados } \\
\hline \multirow{2}{*}{$\mathrm{CTABr}$} & & $\mathrm{C}_{16} \mathrm{Pr}_{3} \mathrm{Br}$ & \\
\hline & & $\mathrm{C}_{16} \mathrm{Et}_{3} \mathrm{Br}$ & \\
\hline \multirow{2}{*}{$\mathrm{CPyBr}$} & & $\mathrm{C}_{16} \mathrm{Me}_{1} \mathrm{Et}_{2} \mathrm{Br}$ & \\
\hline & $\mathrm{Br}^{-}$ & CADMABr & \\
\hline \multirow{2}{*}{$\mathrm{C}_{16} \mathrm{Me}_{2} \mathrm{Et}_{1} \mathrm{Br}$} & & CMImBr & \\
\hline & $\mathrm{Br}^{-}$ & CVImBr & \\
\hline
\end{tabular}

A Concentração Micelar Crítica (CMC) dos surfactantes catiônicos citados foram obtidas através do método da condutividade elétrica. Dessa forma, a condutividade das dispersões de cada surfactante foi medida em diferentes concentrações para uma dada temperatura. Os experimentos foram realizados sob temperatura controlada de $25^{\circ} \mathrm{C}, 40^{\circ} \mathrm{C}$ ou $50^{\circ} \mathrm{C}$, utilizando um banho termoestático. $\mathrm{O}$ método consiste em preparar uma dispersão de alta concentração de surfactante e com o auxílio de uma bureta gotejá-la em um reator encamisado com um volume inicial de água, sob agitação contínua e acoplado no banho termoestático. Dessa forma a concentração no reator é aumentada gradativamente e sua condutividade é medida, utilizando um condutivímetro, em cada concentração. Em seguida, os dados obtidos experimentalmente foram plotados em um gráfico de condutividade elétrica versus concentração molar de surfactante. Trataram-se tais dados utilizando dois métodos distintos: O primeiro foi realizando o ajuste dos dados obtidos em um gráfico com duas retas e identificando o ponto de intersecção que corresponde à CMC, segundo Eising et al., 2008; O segundo método consistiu em ajustar os dados obtidos de condutividade elétrica por concentração molar utilizando a Equação 1 de Carpena et al. (2002), A partir desta, encontrou-se a CMC de cada um dos surfactantes catiônicos.

$$
K_{[\text {Surf }]}=K_{0}+S_{1}[\operatorname{Surf}]+d x\left(S_{2}-S_{1}\right) \ln \left(\frac{1+e^{\frac{([\operatorname{Surf}]-c m c)}{d x}}}{1+e^{-\frac{c m c}{d x}}}\right)
$$

Sendo: $\mathrm{K}$ [surf] = condutividade da solução; $K o$ = condutividade à diluição infinita; $S_{1} \mathrm{e}$ $\mathrm{S}_{2}=$ os coeficientes angulares das retas antes e depois da CMC, respectivamente; e $\mathrm{dx}=$ largura da transição.

Determinou-se também a fração de contra-íons dissociados $(\alpha)$ utilizando o método apresentado por Frahm et al. (1980), no qual os coeficientes angulares $\left(S_{1}\right.$ e $\left.S_{2}\right)$ das duas retas obtidas no ajuste dos pontos experimentais são relacionados utilizando a Equação 2. 


$$
\alpha=\frac{S_{2}}{S_{1}}
$$

\section{RESULTADOS E DISCUSSÃO}

A partir da concentração micelar crítica (CMC), os surfactantes se associam espontaneamente em agregados de menor energia (micelas), de acordo com Maniasso, 2001.Cada surfactante possui uma CMC característica em uma dada temperatura, ou seja, uma concentração mínima para a primeira micela ser formada na dispersão. Obteve-se as CMC de cada surfactante catiônico em três temperaturas distintas $\left(25^{\circ} \mathrm{C}, 40^{\circ} \mathrm{C}\right.$ e $\left.50^{\circ} \mathrm{C}\right)$. A Tabela 2 apresenta esses resultados obtidos pelo método descrito por Carpena et al. (2002). Foi possível constatar através dos dados apresentados na Tabela 2 que o aumento da temperatura implicou no aumento da CMC para todos os surfactantes em questão.

Tabela 2 - CMC dos surfactantes obtida pelo método de Carpena et al. (2002).

\begin{tabular}{|c|c|c|c|c|c|c|c|c|c|}
\hline & CTA & C16Et3 & C16Pr3 & CPy & CMIm & CVIm & CADMA & C16Me2Et1 & C16Me1Et2 \\
\hline $25^{\circ} \mathrm{C}$ & 0,92 & 0,86 & 0,53 & 0,69 & 0,67 & 0,50 & 0,73 & 0,85 & 0,83 \\
\hline $40^{\circ} \mathrm{C}$ & 1,10 & 0,94 & 0,59 & 0,80 & 0,78 & 0,61 & 0,86 & 1,00 & 0,90 \\
\hline $50^{\circ} \mathrm{C}$ & 1,27 & 1,05 & 0,63 & 0,98 & 0,85 & 0,73 & 1,01 & 1,16 & 1,09 \\
\hline
\end{tabular}

As Figuras 1a e 1b apresentam os gráficos contendo os valores experimentais de condutividade elétrica (em temperaturas variadas) por concentração dos surfactantes brometo de cetiltrimetilamônio ( $\mathrm{CTABr}$ ) e do brometo de cetilalildimetilamônio (CADMABr), respectivamente. As curvas da Figura 1 apresentam o perfil característico para esse sistema (dispersão de surfactantes catiônicos). Ou seja, apresentam duas regiões com comportamento linear e a região de transição entre tais regiões.

Figura 1 - Condutividade elétrica $(\mu \mathrm{S} / \mathrm{cm})$ por concentração molar $(\mathrm{mmol} / \mathrm{L})$ das dispersões aquosas de: (a) CTABr; (b) CADMABr.
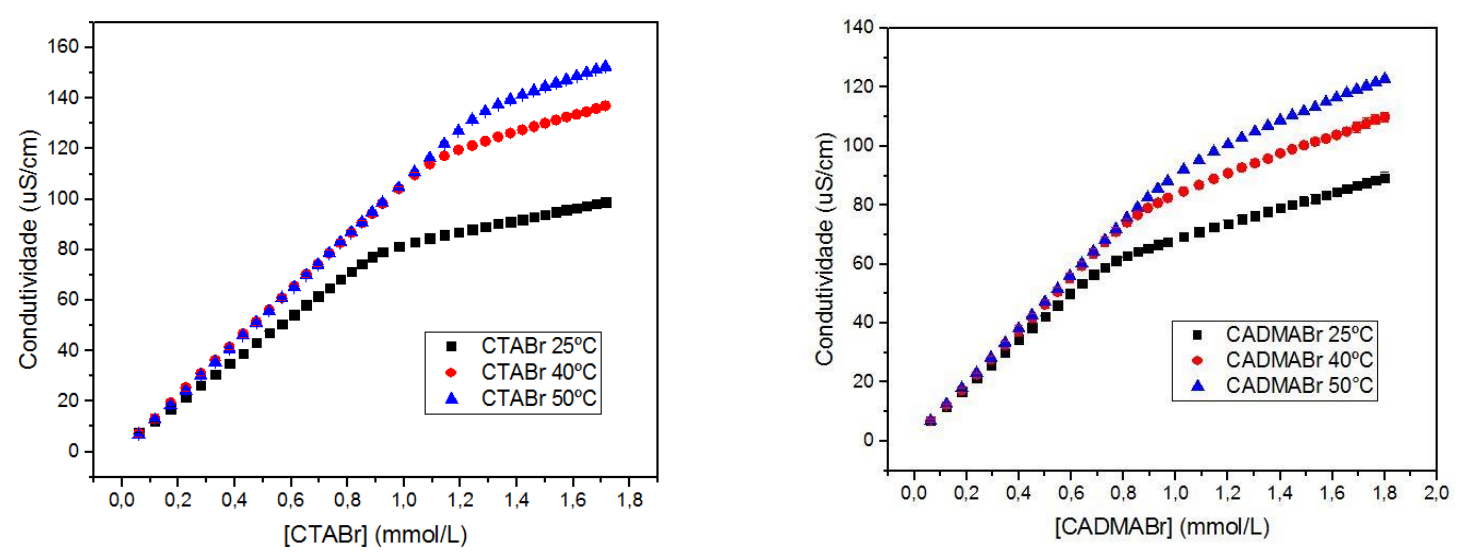

Para a análise da influência do tamanho da cabeça do surfactante e sua CMC, eles foram divididos em três grupos (surfactantes de cabeça simétrica (Grupo 1), cabeça cíclica (Grupo 2), e cabeça assimétrica (Grupo 3)). A Tabela 2 demonstra os resultados adquiridos para cada grupo, juntamente com os valores da fração de contra-íons dissociados $(\alpha)$. 
Tabela 2 - Massa molar dos surfactantes e resultados obtidos utilizando os métodos das equações das retas ou método de Carpena et al. (2002).

\begin{tabular}{|c|c|c|c|c|}
\hline Surfactante & $\begin{array}{c}\text { Massa Molar } \\
{[\mathrm{g} / \mathrm{mol}]}\end{array}$ & $\begin{array}{c}\text { CMC (Eq. Retas) } \\
{[\mathrm{mmol} / \mathrm{L}]}\end{array}$ & $\begin{array}{c}\text { CMC (Carpena) } \\
{[\mathrm{mmol} / \mathrm{L}]}\end{array}$ & $\alpha$ \\
\hline \multicolumn{5}{|c|}{ Grupo 1 } \\
\hline CTABr & 364,45 & 0,92 & 0,92 & 0,27 \\
\hline C16Et3Br & 406,53 & 0,86 & 0,86 & 0,38 \\
\hline C16Pr3Br & 448,61 & 0,56 & 0,53 & 0,45 \\
\hline \multicolumn{5}{|c|}{ Grupo 2 } \\
\hline CPyBr & 384,44 & 0,69 & 0,69 & 0,30 \\
\hline CMImBr & 378,47 & 0,67 & 0,67 & 0,30 \\
\hline CVImBr & 392,50 & 0,52 & 0,50 & 0,31 \\
\hline \multicolumn{5}{|c|}{ Grupo 3 } \\
\hline CADMABr & 390,48 & 0,75 & 0,73 & 0,32 \\
\hline C16Me2Et1Br & 378,47 & 0,85 & 0,86 & 0,29 \\
\hline C16Me1Et2Br & 392,50 & 0,83 & 0,83 & 0,33 \\
\hline
\end{tabular}

Os surfactantes catiônicos de cabeça simétrica (Grupo 1) e os de cabeça cíclica (Grupo 2) seguiram uma tendência em relação a massa molar dos surfactantes: quanto maior a massa molecular, menor a CMC do surfactante. Devido ao fato dos surfactantes com cabeças polares de maiores volumes, possuírem menor densidade de carga superficial, dessa forma a repulsão é menor e menor a CMC. No entanto, o grupo de surfactantes de cabeça assimétrica (Grupo 3) não seguiu a mesma tendência, isto se deve a comparação ter sido realizada pela massa molecular dos surfactantes, a qual não é sempre proporcional ao volume destes. Em relação as CMCs obtidas pelo método das equações das retas e pelo método de Carpena, os resultados obtidos para os dois tratamentos não possuem grandes discrepâncias entre si. No entanto o segundo método é mais preciso, uma vez que considera a CMC como uma faixa de transição.

\section{CONCLUSÕES}

O aumento da temperatura das dispersões dos surfactantes catiônicos dificultou a formação de micelas, isto deve-se ao maior grau de agitação das moléculas que dificultam a estabilização na forma micelar. Além disso, em maiores temperaturas a cauda apolar é menos hidratada, e dessa forma o efeito hidrofóbico tem menor impacto no valor da CMC. Os surfactantes com maior volume de cabeças hidrofóbica possuem carga mais distribuída e menor densidade de carga superficial, dessa forma há menor repulsão entre as moléculas, e maior facilidade de estabilização da micela e, portanto, menor valor de CMC. Ademais, a massa molecular dos surfactantes foi utilizada no trabalho para estabelecer uma relação entre as CMC obtidas, entretanto a relação da massa molecular e volume da cabeça do surfactante não foi sempre proporcional.

\section{REFERÊNCIAS}

CAMPOS, A. F. P. Síntese e propriedades de sílicas híbridas contendo surfactantes catiônicos Tese de doutorado. Universidade Federal de São Carlos, São Carlos, 2015. 
CARPENA, P.; AGUIAR, J.; BERNAOLA-GALVÁN P.; RUIZ, C. C. Problems Associated with the Treatment of Conductivity-Concentration Data in Surfactant Solutions: Simulations and Experiments. Langmuir, Vol. 18, n. 16, p.6054-6058, 2002.

EISING, R.; MORÉS, S.; BELLETTINI, I. C.; FELIPPE, A. C.; DAL-BÓ, A. G.; ZANETTE, D. Formação de micelas mistas entre o sal biliar colato de sódio e o surfactante aniônico dodecanoato de sódio. Quim. Nova, Vol. 31, No. 8, 2065-2070, 2008.

FABIANO, D. P. Síntese e avaliação de sílica tipo M41S contendo cátions CTA em transesterificação catalítica. Tese de doutorado. Departamento de Engenharia Química, Universidade Federal de São Carlos. São Carlos, 2010a.

FRAHM, J.; DIEKMANN, S.; HAASE, A. Electrostatic properties of ionic micelles in aqueous solutions. Berichte de Bunsen-Gesellschaft, v.84, n.6, p.566-571, 1980.

GALGANO, P. D.; Líquidos Iônicos Tensoativos: Correlação entre Estrutura Molecular e Propriedades Micelares de Cloretos de 1,3-dialquilimidazólio. Tese de doutorado. Instituto de Química, Univ. São Paulo. São Paulo, 2012.

GALGANO, P. D.; EL SEOUD, O. A. Micellar properties of surface active ionic liquids: A comparison of 1-hexadecyl- 3-methylimidazolium chloride with structurally related cationic surfactants. Journal of Colloid and Interface Science v. 345, n.1, p.1-11, 2010.

GEORGOGIANNI, K.G.; A.P. Katsoulidis; P.J. Pomonis; M.G. Kontominas, Fuel Processing Technology, 2009.

GRECCO, S.T.F.; RANGEL, M.C.; URQUIETA-GONZÁLEZ, E.; Zeólitas hierarquicamente estruturadas. Química Nova, v. 36, p. 131-142, 2013.

KUBOTA, Y.; NISHIZAKI, Y.; IKEYA, H.; SAEKI, M.; HIDA, T.; KAWAZU, S.; YOSHIDA, M.; FUJII, H.; SUGI, Y. Organic-silicate Hybrid Catalysts Based on Various Defined Structures for the Knoevenagel Condensation, Microporous and Mesoporous Materials, 70, 135-149, 2004.

MANIASSO, N. Ambiente Micelares em Química Analítica. Quim. Nova, Vol. 24, n.1, p.8793, 2001.

OSELIERO FILHO, P.L.; Estudo estrutural e termodinâmico de sistemas auto - organizados: Micelas em solução. Dissertação de mestrado. Instituto de Física, Univ. de São Paulo. São Paulo, 2013.

QUINTELLA, C.M.; TEIXEIRA, L.S.G.; et al., Cadeia do biodiesel da bancada à indústria: uma visão geral com prospecção de tarefas e oportunidades para P\&D\&I, Química Nova, 2009.

SILVA, L. L. Síntese da sílica MCM-41 usando um surfactante catiônico polimerizável e avaliação na transesterificação catalítica. Dissertação de Mestrado. Departamento de Engenharia Química, Universidade Federal de São Carlos. São Carlos, 2014. 